\title{
XUtils Ver. 1: A Set of Gatan DigitalMicrograph Plug-ins for Characterization of XEDS-detector Performance Parameters in an AEM
}

\author{
M. Watanabe ${ }^{1}$ \\ ${ }^{1}$ Dept of Materials Science and Engineering, Lehigh University, Bethlehem. PA 18015, USA.
}

Before performing practical X-ray analysis by X-ray energy dispersive spectrometry (XEDS) in an analytical electron microscope (AEM), it is essential to figure out fundamental detector performance parameters, such as energy resolution, peak-to-background ratio, detector collection angle, etc. Some detector parameters should be measured at least once when the system is installed or significantly upgraded. Other parameters also need to be measured time to time to monitor the system performance in a consistent manner. Fortunately, most of the XEDS characteristics can be measured using Egerton's NiOx test specimen [1-3]. To carry these measurements out routinely and consistently, a set of plug-ins for Gatan DigitalMicrograph named XUtils was developed. In the XUtils plug-ins, two main plug-ins can be used for determination of detector performance parameters as briefly described below.

NiOx: The main dialog is shown in Fig. 1(a). This plug-in offers determination of energy resolution, peak-to-background (P/B) ratio, inverse hole-count (IHC), Mo K/L ratio, detector collection angle and detector efficiency using a single spectrum from the NiOx test specimen. For example, Fig. 1(b) shows a result of Gaussian fitting to the Ni K $\alpha$ peak for determination of the energy resolution. The system energy resolution defined at $5.9 \mathrm{keV}$ is converted from the full-width at half-maximum value of the $\mathrm{Ni} \mathrm{K} \alpha$ peak as proposed by Bennett and Egerton [2]. The P/B ratio and IHC are useful to evaluate the AEM-XEDS interface in an AEM [4]. Both the parameters can be determined from a single NiOx spectrum. Figure 1(c) shows a plot of P/B ratio against IHC, measured in three different instruments operated at 100, 200 and $300 \mathrm{keV}$, respectively. Since higher values are preferable in both parameters to perform better X-ray analysis in an AEM, instruments laid at top right corner should be superior in X-ray analysis.

NiOxIceC: This plug-in determines the accumulated ice and carbon thicknesses at the detector window from two spectra measured in different time period from the NiOx test specimen, based

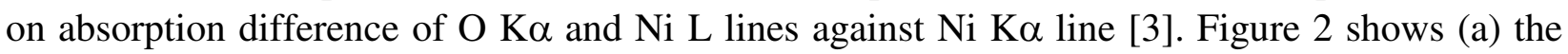
main dialog of the plug-in and (b) a comparison of two X-ray spectra from the NiOx film acquired in a same instrument at different time period (one was recorded after detector conditioning and other was a few month later). In this particular case, the accumulated ice and carbon layers are $1.67 \pm 0.04 \mu \mathrm{m}$ and $53.1 \pm 8.5 \mathrm{~nm}$ with $99 \%$ confidence limit, respectively.

In addition to the above plug-ins, this package contains a function to import a spectrum in the EMSA/MAS file format, which is the most commonly used in electron microscopy community and supported by most XEDS manufactures in their systems. Therefore, as long as the spectrum is saved in the EMSA format, it can be converted into DigitalMicrograph and the detector parameters can be determined via the above plug-ins. This plug-in package is freely available through this author's home page [5]. The installation procedure and detailed usage can be found in the help file comes with the plugin package. To run any functionalities in this plug-in package, obviously Gatan DigitalMicrograph is required. 


\section{References}

[1] R.F. Egerton \& S.C. Cheng, Ultramicrosc. 55 (1994), 43.

[2] J.C. Bennett \& R.F.Egerton, J. Microsc. Soc. Am., 1 (1995), 143.

[3] K. Wong \& R.F. Egerton, Proc. Microscopy and Microanalysis 1995, Jones and Begell Publishing New York (1995), 600.

[4] C.E. Lyman, et al., J. Microsc. 176 (1994), 85.

[5] M. Watanabe, (2009), XUtils - X-ray analysis utilities for Gatan DigitalMicrograph, available from Department of Materials Science \& Engineering, Lehigh Univeristy, Bethlehem, PA18015 (http://www.lehigh.edu/ maw3/research/xutilmain.html).

[6] The author wish to acknowledge the financial support of the NSF through grant (DMR-0804528).

Fig. 1 (a)

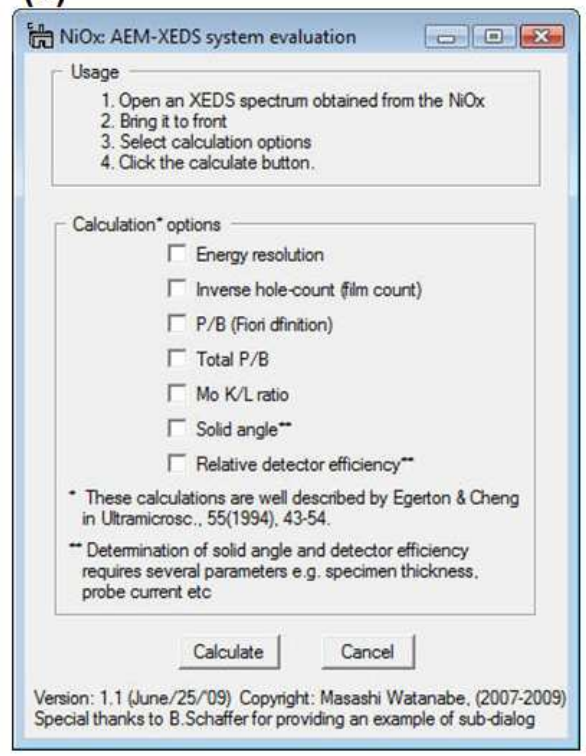

Fig. 2

(a)

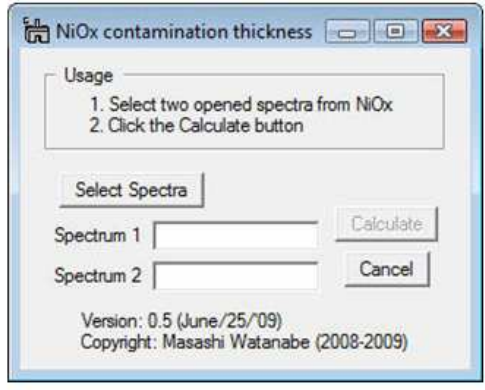

(b)
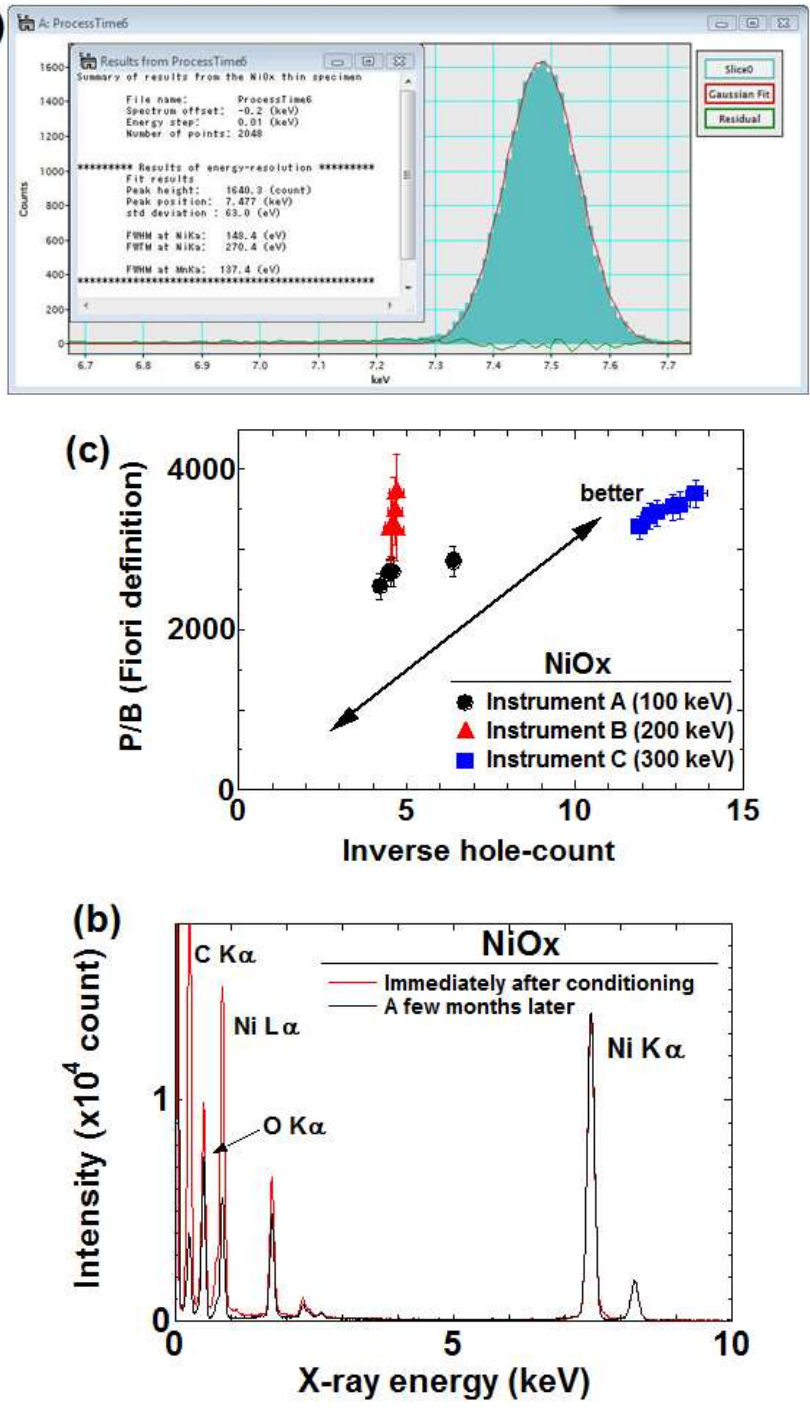

Figure 1: (a) Main dialog of the NiOx plug-in package, (b) an example of energy resolution determination using the $\mathrm{Ni}$ Ka peak from the $\mathrm{NiOx}$ test specimen. and (c) plots of the $\mathrm{P} / \mathrm{B}$ ratio against IHC, measured from three different instruments operated at 100, 200 and $300 \mathrm{keV}$. Figure 2 (a) Main dialog of the $\mathrm{NiOxIceC}$ plug-in package and (b) a comparison of X-ray spectra obtained from the same $\mathrm{NiOx}$ test specimen in a same instrument at different time period. 